
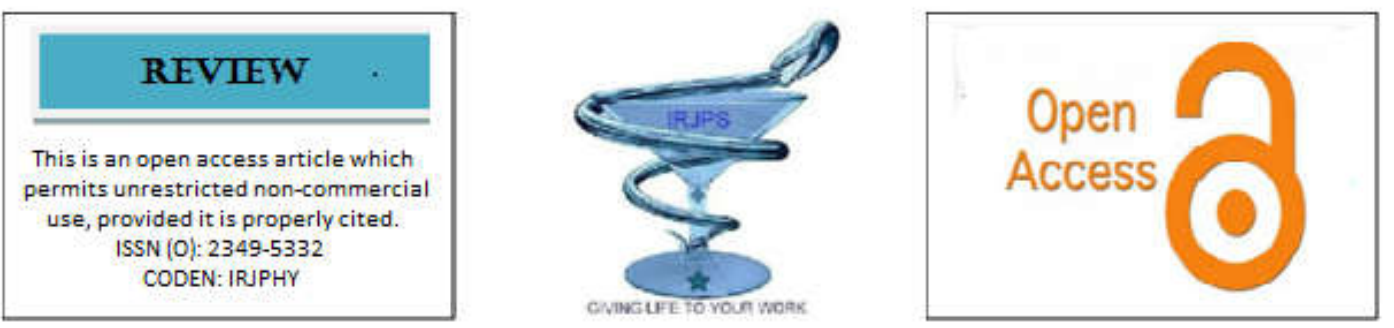

\title{
S.O.M.A. - A NEW ERA FOR DIABETIC PATIENT
}

Dibyendu Shil ${ }^{* 1}$, Tanmay Sarma ${ }^{2}$, Masud Reja ${ }^{1}$, Amit Samanta ${ }^{1}$

${ }^{1}$ Mata Gujri College of Pharmacy, Kishanganj, Bihar, India

${ }^{2}$ Girijananda Chowdhury Institute of Pharmaceutical Science, Guwahati, Assam, India

\begin{abstract}
:
Diabetes mellitus which is a metabolic disorder could be a very fatal disease within a very few years. Recently, a group of researchers from M.I.T., Harvard Medical School, Brigham Women's Hospital and Novo Nordisk pharmaceutical company has developed a self-orienting millimeter- scale applicator (SOMA) for oral delivery of insulin. They have developed a capsule look like a 'Leopard Tortoise' shell shape that is designed to have one needle covered with a shaft, allowing them to inject the drugs only into the interior lining of stomach, where they would be broken down by stomach acids before having any effect. The stomach wall has no pain receptors, so the researchers believe that the patient would not be able to feel the injection. This is a new technology that could benefit many patients. The motivation of this invention is to make it easier for patients to take medication, particularly medications that require an injection.
\end{abstract}

KEY WORDS: SOMA, Insulin, Diabetes mellitus.

Corresponding author- Dr. Dibyendu Shil Email id: drdibyendushil@gmail.com Ph no: 7002883942
Indian Research Journal of Pharmacy and Science; 26(2020)2411-2414; Journal Home Page: https://www.irjps.in DOI: 10.21276/irjps.2020.7.4.2 


\section{INTRODUCTION:}

Diabetes mellitus is a group of metabolic disorders in which there is high blood sugar level over a prolonged period. Symptoms of high blood sugar include frequent urination, increased thirst and increased hunger. For many people with diabetes injecting insulin in multiple times per day is the only way to control the blood glucose level. Daily injection can cause discomfort and raw attention, formulations require refrigeration and they generate bio hazardous needle waste and these cause worst health outcome ${ }^{1}$. Recently an MIT-led research team has developed a Self Orienting Millimeter Scale Actuator (SOMA) capsule (Figure 1 and 2) that could be used to deliver oral doses of insulin ${ }^{3}$, potentially replacing the injections that people with type I diabetes have to give themselves every day. About the size of a blue berry, the capsule contains a small needle made of compressed insulin, which is injected after the capsule reaches the stomach. During the test, the researchers showed that they could deliver enough insulin to lower blood sugar to levels comparable to those produced by injection given through skin. The tip of the needle is made of nearly 100 percent compressed, freeze dried insulin, using the same process used to form tablets. The shaft of the needle, which does not enter the stomach wall, is made from another biodegradable material. The research team also includes scientist from the pharmaceutical company Novo Nordisk ${ }^{2}$.

\section{Figure 1: SOMA with respect to Leopard tortoise}

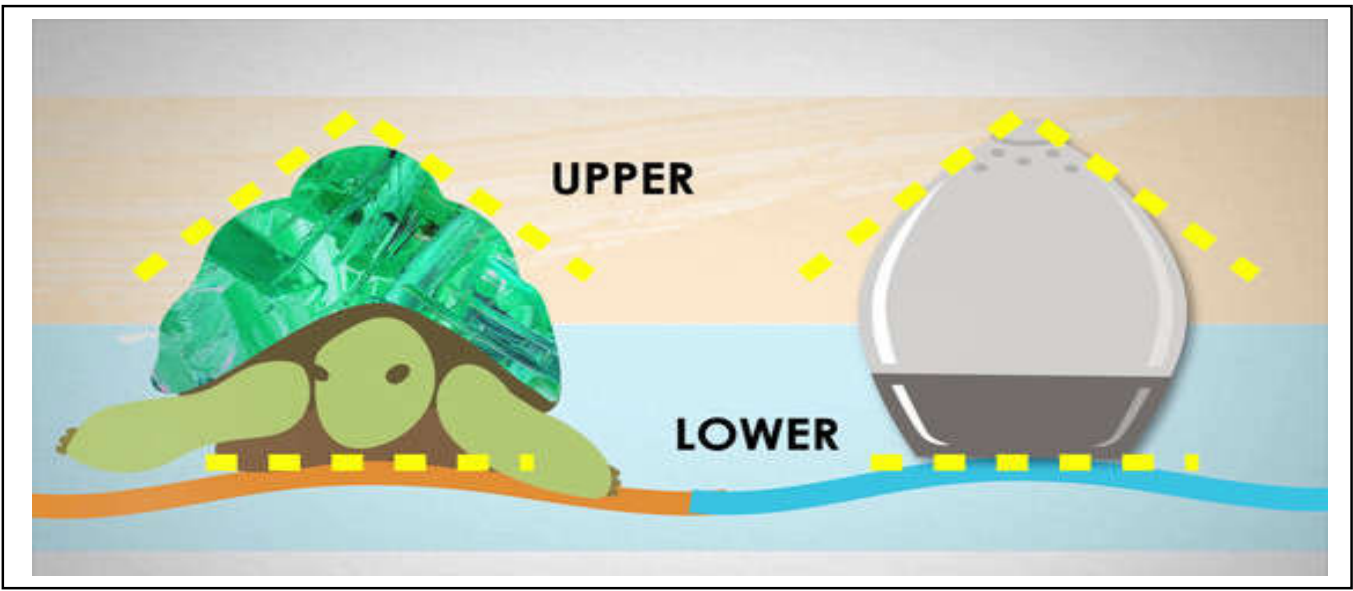

Figure 2: Capsule design

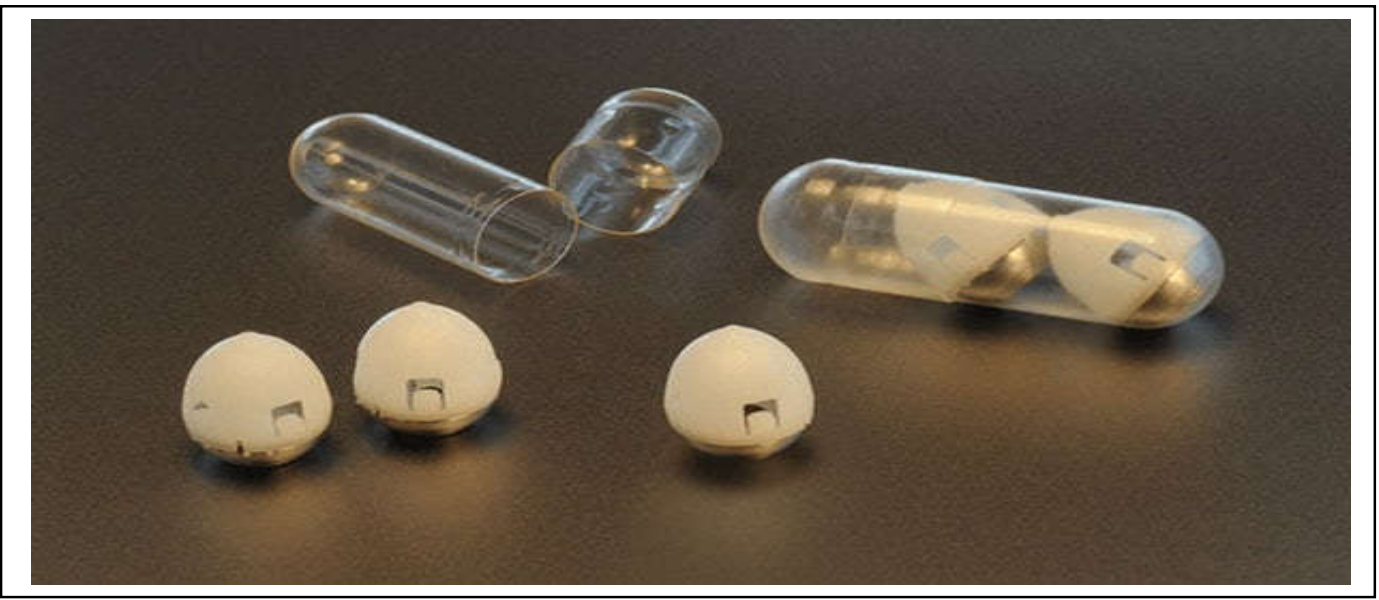




\section{MECHANISM:}

In this capsule, the researchers make the design to have just one needle, allowing them to avoid injection drugs into the interior of the stomach, where they would be broken down by stomach acids before having any effect ${ }^{1}$. Within the capsule, the needle is attached to a compressed spring that is held in place by a disk made of sugar. When the capsule is swallowed, water in the stomach dissolves the sugar disk, releasing the spring and injecting the needle into the stomach wall. SOMA arrives at the stomach in 1 second after swallowing, the injection needle jumps out in 1 minute and all insulin is designed to be completely released into the blood stream in 1 hour (Figure 3$)^{3}$. The researchers drew their inspiration for the self orientation feature from a tortoise known as leopard tortoise. This tortoise has the capability to right itself it roll onto its back. The researchers used computer modeling to come up with a variant of this shape for their capsule, which allows it to reorient itself even in the dynamic environment of the stomach. The team designs a novel system with the ability to consistently orient and release the drug into tissue wall. The self orienting millimeter scale Actuator known as SOMA consistently deliver insulin with and efficiency comparable to injection in the body ${ }^{3}$. Both the tortoise shell and SOMA possess upper portion with pointed tops and lower portion with the level bottoms ${ }^{3}$. The high curvature body allows the device to reorient itself utilizing gravity alone the flat base ensure that SOMA does not tip over when moved by stomach contraction. The stomach walls muscular tissue act as a natural barrier and prevents the risk of perforation during drug insertion. The stomach wall has no pain receptors, so the researchers believe that patient would not be able to feel the injection. ${ }^{4}$ Once the tip of needle injected into the stomach wall, the insulin dissolves at a rate that can be controlled by the researchers as the capsule is prepared. In this study it took about an hour for all of the insulin to be fully released into the bloodstream ${ }^{3}$. After the capsule releases its contents, it can pass harmlessly through the digestive system. The researchers found no adverse effects from the capsule, which is made from biodegradable polymer and stainless steel components.

\section{Figure 3: Time taken by capsule to dissolve in blood stream}

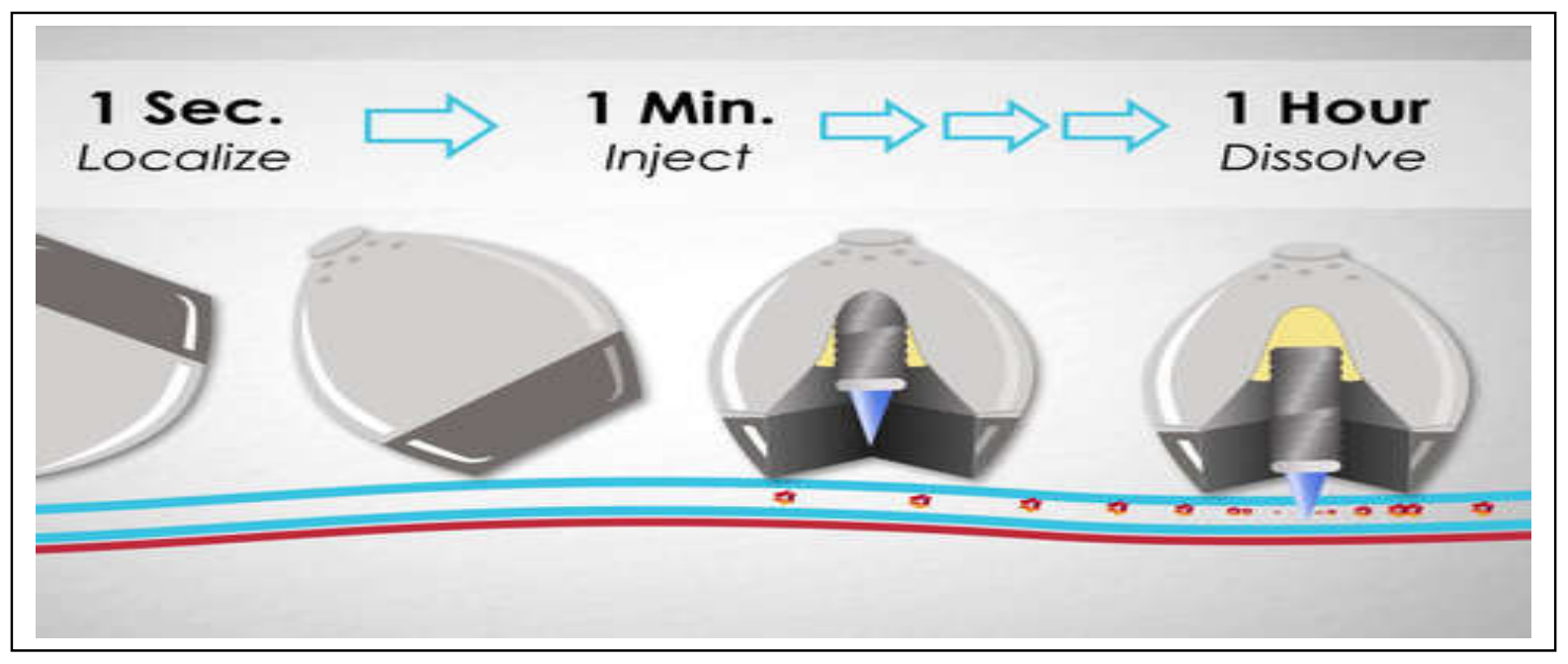




\section{DISCUSSION:}

The Self Orienting Millimeter Scale Actuator (SOMA) plays important role in this mechanism of drug delivery ${ }^{3}$. It helps the capsule to stay in the contact with the stomach lining, so that proper and efficient drug delivery can occur. In test in pigs the researchers showed that they could successfully deliver upto 300 microgram of insulin. More recently they have been able to increase the dose to 5 milligram which is comparable to the amount that a patient with type I diabetes would need to inject. The shaft which covers the content of the capsule also made up of biodegradable content. This is not harmful for the patient. Patient does not require to take injections multiple times per day, which makes discomfort to the patient. The high curvature body allows the device to reorient itself utilizing gravity alone. The flat base ensures that the SOMA does not tip over when moved by stomach contraction ${ }^{4}$. The rate of the drug release at stomach lining can be measured before the drug administration. Patient can not feel any kind of pain, because the stomach wall does not contain any kind of pain receptor.

\section{CONCLUSION:}

Hopefully this new technology will be capable to provide new era for diabetic patient. It make easier for patient to take medication especially medication that require injection. The MIT team is continuing working with Novo Nordisk to further develop the technology and optimize the manufacturing process for the capsule.

\section{REFERENCE:}

1. Gerardo PC, Mathiowitz E, Oral insulin delivery, 2017; 3(18): 249-257

2. Yasmeen, Mamatha T, Md Zubair, Begum S, Various Emerging Trends in insulin Drug Delivery System, 2018; 5(23): 234-239.

3. Anne T, MIT News office; New pill can deliver insulin, February 7, 2019

4. Kanchan GC, Mukadam A, Jahagirdar KH, Shaikh SS, Bhise KS, Advances in insulin delivery devices, 2009; 2(9): 1515-1520.

CONFLICT OF INTEREST REPORTED: NIL; SOURCE OF FUNDING: NONE REPORTED 